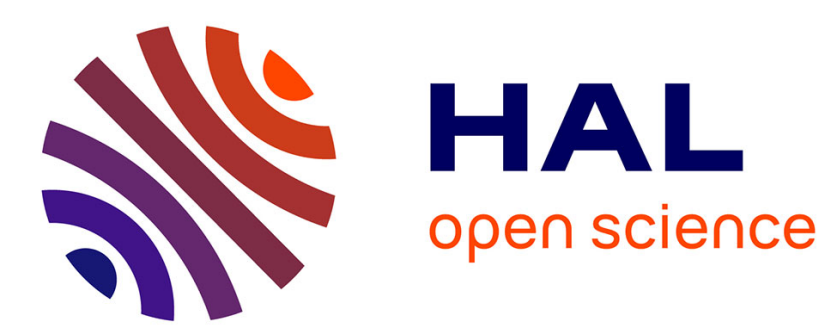

\title{
CHARGE DENSITY WAVE PHASE TRANSITIONS OBSERVED BY X-RAY PHOTOEMISSION
}

\author{
R. Pollak, H. Hughes
}

\section{To cite this version:}

R. Pollak, H. Hughes. CHARGE DENSITY WAVE PHASE TRANSITIONS OBSERVED BY X-RAY PHOTOEMISSION. Journal de Physique Colloques, 1976, 37 (C4), pp.C4-151-C4-155. 10.1051/jphyscol:1976423 . jpa-00216537

\section{HAL Id: jpa-00216537 https://hal.science/jpa-00216537}

Submitted on 1 Jan 1976

HAL is a multi-disciplinary open access archive for the deposit and dissemination of scientific research documents, whether they are published or not. The documents may come from teaching and research institutions in France or abroad, or from public or private research centers.
L'archive ouverte pluridisciplinaire HAL, est destinée au dépôt et à la diffusion de documents scientifiques de niveau recherche, publiés ou non, émanant des établissements d'enseignement et de recherche français ou étrangers, des laboratoires publics ou privés. 


\title{
CHARGE DENSITY WAVE PHASE TRANSITIONS OBSERVED BY X-RAY PHOTOEMISSION
}

\author{
R. A. POLLAK and H. P. HUGHES (*) \\ IBM Thomas J. Watson Research Centre, Yorktown Heights, \\ New York 10598, U. S. A.
}

\begin{abstract}
Résumé. - Nous avons mesuré les énergies de liaison des niveaux $4 \mathrm{f}$ du Tantale dans $\mathrm{TaS}_{2}$, $\mathrm{TaSe}_{2}$ et $\mathrm{Ta}_{0,97} \mathrm{Hf}_{0,03} \mathrm{~S}_{2}$ entre $14 \mathrm{~K}$ et $371 \mathrm{~K}$ avec la photoémission à rayon X (XPS). La présence d'onde de densité de charge (CDW's) a pour effet de produire des potentiels non équivalents aux différents sites des atomes Ta. Ceci entraîne le dédoublement des lignes $4 \mathrm{f}$ en XPS ; l'écartement est une mesure de l'amplitude de l'onde de densité de charge, et représente un paramètre d'ordre pour le système CDW-réseau. La forme des composants de l'émission XPS permet de déduire la configuration de la CDW vis-à-vis du réseau, et puisque les gradients de potentiels aux sites non équivalents des atomes Ta sont différents, la largeur des composants fournit une mesure de la nature dynamique du système CDW-réseau. Les résultats démontrent l'existence d'une fluctuation CDW dans la phase où il y a coïncidence entre le réseau et la $\mathrm{CDW}$. Dans les cas où la coïncidence entre le réseau et la CDW est imparfaite, ou qu'il n'y en a pas, les CDW ne peuvent garder la cohérence à longue distance. Dans le $1 \mathrm{~T} \mathrm{Ta} \mathrm{Ta}_{0,} \mathrm{Hf}_{0,03} \mathrm{~S}_{2}$, les atomes de Hafnium ont pour effet d'ancrer les CDW (qui ne sont pas en coïncidence avec le réseau) en domaines.
\end{abstract}

\begin{abstract}
X-ray photoemission (XPS) measurements of the binding energies of the Ta $4 \mathbf{f}$ core levels in $\mathrm{TaS}_{2}, \mathrm{TaSe}_{2}$, and $\mathrm{Ta}_{0.97} \mathrm{Hf}_{0.03} \mathrm{~S}_{2}$ at temperatures between $14 \mathrm{~K}$ and $371 \mathrm{~K}$ are reported. The presence of charge density waves (CDW's) produces inequivalent potentials at different $\mathrm{Ta}$ sites which split the $4 \mathrm{f}$ XPS lines; the splitting is a measure of the CDW amplitude and represents an order parameter for the CDW-lattice system. The form of the components of the split XPS lines allows the CDW-lattice configuration to be deduced, and since the potential gradients at inequivalent $\mathrm{Ta}$ sites are different, the unequal widths of the components yields a measure of the fluctuating nature of the CDW-lattice system. The results indicate the existence of CDW fluctuations in commensurate phases, and that in some incommensurate and quasi-commensurate phases the CDW's cannot maintain long range coherence. In $1 \mathrm{~T}-\mathrm{Ta}_{0} ;{ }_{97} \mathrm{Hf}_{0} ; 0{ }_{3} \mathrm{~S}_{2}$ the $\mathrm{Hf}$ impurities pin the incommensurate CDW's into domains.
\end{abstract}

1. Introduction. - Charge density waves (CDW's) arise from anomalies in the dielectric susceptibility which occur when large areas of the Fermi surface are spanned by the same wavevector. This condition is more easily satisfied in one-and two-dimensional materials than in general, so metallic layered materials are excellent candidates for observing CDW effects. In particular the $1 \mathrm{~T}$ and $2 \mathrm{H}$ polytypes of $\mathrm{TaS}_{2}$ and $\mathrm{TaSe}_{2}$ have attracted considerable attention; the periodic lattice distortions associated with the CDW's have been extensively studied by electron [1-5] and neutron diffraction [6], and CDW-induced phase transformations are clearly observed in electrical resistivity measurements $[1]\left({ }^{1}\right),\left({ }^{2}\right)$.

The existence of periodic variations in the conduction charge density implies that crystal lattice sites which are identical in the absence of CDW's experience different local charge environments i. e. become inequivalent. The local charge environment causes a

(*) Permanent address: Cavendish Laboratory, Madingley Road, Cambridge, U. K.

(1) Penney, T. (1976) private communication.

(2) Craven, R. A. (1976) private communication. chemical shift of the atomic core level binding energies, so it is possible in principle to distinguish inequivalent sites through X-ray photoelectron spectroscopy (XPS). Being very sharp and well resolved, the spin-orbit split Ta $4 \mathrm{f} \frac{7}{2}$ and $4 \mathrm{f} \frac{5}{2}$ core lines form excellent probes for the local charge density in $1 \mathrm{~T}$ and $2 \mathrm{H} \mathrm{TaS}$ and $\mathrm{TaSe}_{2}$. Observations of CDW effects on the Ta 4f XPS spectra have been reported by Wertheim, Di Salvo and Chiang [7] and a preliminary discussion of some of the present results has already been given [8].

Details of experimental techniques, sample growth, and data analysis methods will be presented elsewhere [9]. Reported here are XPS measurements on the $\mathrm{Ta}$ 4f core lines for $1 \mathrm{~T}-\mathrm{TaSe}_{2}, 1 \mathrm{~T}-\mathrm{TaSe}_{2}$, $1 \mathrm{~T}-\mathrm{Ta}_{0.97} \mathrm{Hf}_{0.03} \mathrm{~S}_{2}$ and $2 \mathrm{H}-\mathrm{TaSe}_{2}$, at temperatures between $\sim 14 \mathrm{~K}$ and $371 \mathrm{~K}$. The spectra were obtained with a modified Hewlett-Packard 5950 A ESCA spectrometer using a monochromated $\mathrm{AlK}_{\alpha} \mathrm{X}$-ray source $(1486 \mathrm{eV})$. Except for $2 \mathrm{H}-\mathrm{TaSe}_{2}, \mathrm{CDW}$ effects can be clearly followed as functions of temperature, and those for $1 \mathrm{~T}-\mathrm{TaS}_{2}$ exhibit the various phase transformations observed in electrical and diffraction 
studies. The results are interpreted in terms of a simple model of the CDW-crystal lattice system, and allow the configuration between the lattice and the CDW's to be deduced. Effects attributable to dynamic fluctuations of the CDW's with respect to the lattice are also observed.

2. Results and Discussion. - $2.11 \mathrm{~T}-\mathrm{TaS}_{2}$. Figure 1 shows the $\mathrm{Ta} 4 \mathrm{f}$ lines for $1 \mathrm{~T}-\mathrm{TaS}_{2}$ at $371 \mathrm{~K}$,

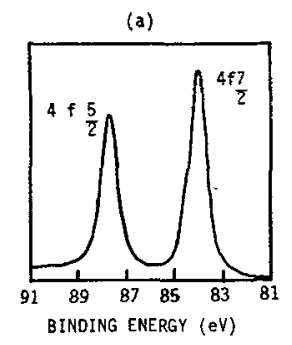

(b)

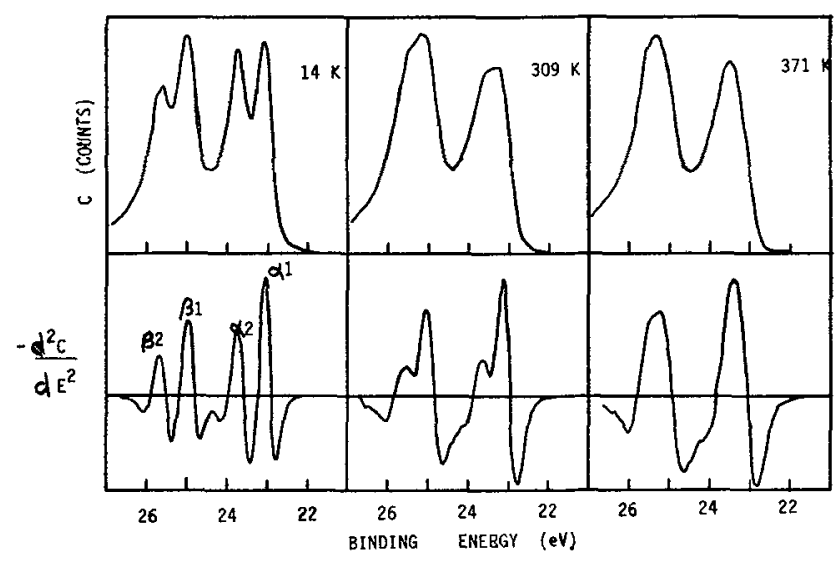

FrG. 1. - a) XPS spectrum of Au $4 \mathrm{f}$ lines from evaporated gold film. b) $\mathrm{Ta} 4 \mathrm{f}$ lines, and their second derivatives, for $1 \mathrm{~T}-\mathrm{TaS}_{2}$ at $14 \mathrm{~K}, 309 \mathrm{~K}$ and $371 \mathrm{~K}$.

$309 \mathrm{~K}$ and $14 \mathrm{~K}$, i. e. on the $1 \mathrm{~T}_{1}, 1 \mathrm{~T}_{2}$ and $1 \mathrm{~T}_{3}$ phases of this material (notation of ref. [4]). Also shown are the $4 f$ lines of elemental gold for comparison, and the negative of the second derivatives of the TaLiF spectra. The Au $4 f$ lines are very sharp (near the instrumental resolution of $\sim 0.5 \mathrm{eV}$ ) and very nearly symmetric ; the relative intensities are in excellent agreement with those expected from their multiplicities $(8: 6)$. In striking contrast are the Ta $4 f$ lines; each line has a long tail running to higher binding energies introducing considerable line asymmetry. This is believed to be a many-body effect, the MahanNozières-de Dominicis (MND) effect, the creation of electron-hole pairs around the Fermi-level during photoemission [10-12].

At $14 \mathrm{~K}\left(1 \mathrm{~T}_{3} \sqrt{13}\right.$ commensurate CDW phase $)$ the lines show a well resolved splitting of $0.69 \mathrm{eV}$; the components $\beta 1$ and $\beta 2$ of the $4 \mathrm{f} \frac{5}{2}$ line are broader than those, $\alpha 1$ and $\alpha 2$, of the $4 \mathrm{f} \frac{7}{2}$ line, presumably a lifetime effect, while $\alpha 2$ is always broader than $\alpha 1$. At $309 \mathrm{~K}\left(1 \mathrm{~T}_{2}\right.$ quasi-commensurate phase in which the CDW wavevectors differ slightly in direction and magnitude from the commensurate condition) the $\mathrm{Ta} 4 \mathrm{f}$ lines differ markedly from those of $\mathrm{Au}$ because there is still a splitting present, resolved in the second derivative but not directly, which increases the apparent linewidths. At $371 \mathrm{~K}\left(1 \mathrm{~T}_{1}\right.$ incommensurate phase) there is no detectable splitting, so the lines are narrower.

The substructure of the Ta $4 \mathrm{f}$ lines is clearly connected with the various CDW phases of this material. This is vividly illustrated in figure 2 where the CDW

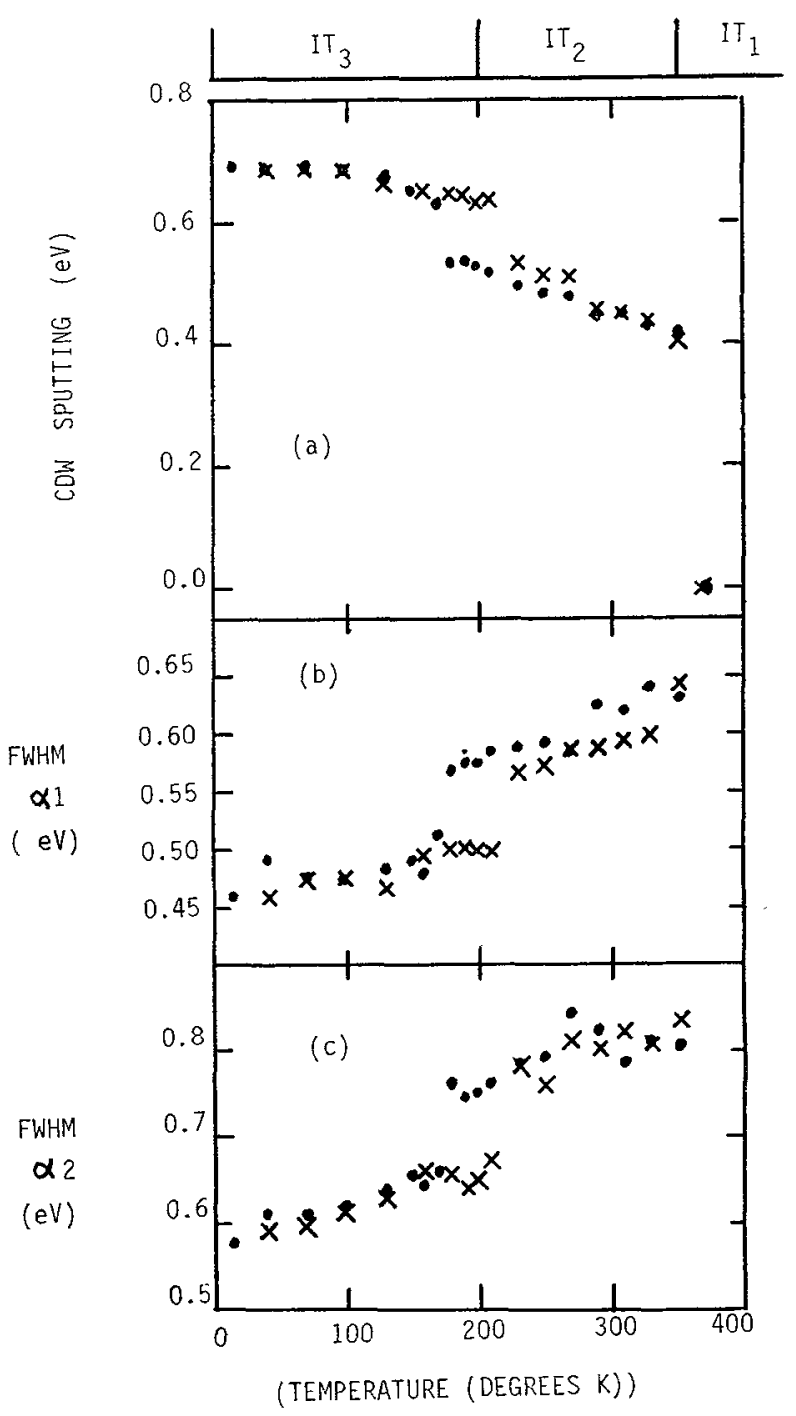

FIG. 2. $-1 \mathrm{~T}-\mathrm{TaS}_{2} \mathrm{Ta} 4 \mathrm{f} \frac{7}{2}$ line. a) $\mathrm{CDW}$ splitting, b) full width half maximum of component $\alpha 1, c)$ full width half maximum of component $\alpha 2$. The symbols $\times$ and . correspond to increasing and decreasing temperature respectively.

induced splitting between $\alpha 1$ and $\alpha 2$, and their widths (obtained by fitting the experimental data with pairs of asymmetric Lorentzian peaks) are plotted as functions of temperature. The hysteresis assoeiated 
with the $1 \mathrm{~T}_{3}-1 \mathrm{~T}_{2}$ transition around $190 \mathrm{~K}$ observed in electrical resistivity is also seen here, and the CDW splitting falls sharply to zero at the $1 T_{2}-1 T_{1}$ transition at $350 \mathrm{~K}$.

The splittings arise from the emission from at least two distinct types of $\mathrm{Ta}$ site having different local charge densities. A simple model of the situation can be constructed by assuming, in a single layer approximation, a rigid lattice (the distortions produced by the CDW's are relatively small) with three sinusoidal plane charge density waves with wavevectors in the layer given by, electron diffraction experiments. The configuration of the CDW's with respect to the lattice is then defined by two parameters; the position $R_{0}$, relative to some Ta site chosen as origin, at which all three waves have some common phase $\varphi$. It is then possible to calculate the local charge distributions at various sites and to construct histograms for the number density of sites with particular local charge. These histograms, convoluted with a broadening factor, then reflect the anticipated XPS spectra. For commensurate CDW's a small discrete number of inequivalent sites is expected, while incommensurate CDW's produce continuous distributions.

For the $\sqrt{13} 1 \mathrm{~T}_{3}$ commensurate condition a $\mathrm{CDW}$ supercell is formed containing $13 \mathrm{Ta}$ atoms; for $\boldsymbol{R}_{\mathbf{0}}=0$ and $\varphi=0$ these 13 sites form a $6: 6: 1$ distribution which might explain the observed splitting of the $\mathrm{Ta} 4 \mathrm{f}$ lines into two components of roughly equal intensity. However there is no evidence for the single extra site emission on the low binding energy side of the peaks $\alpha 1$ and $\alpha 2$, while it could be concealed in the MND tail, so reversing the phase to $\varphi=180^{\circ}$ to give a $1: 6: 6$ distribution seems reasonable. No other simple configuration gives two peaks of roughly equal intensity as observed. In this case all three CDW's produce a minimum of electronic charge around a single Ta site, emission from which is therefore at higher binding energy than for other sites in the CDW supercell.

Applying this simple approach to the $1 \mathrm{~T}_{2}$ quasicommensurate phase with an infinite two-dimensional array of Ta sites produces distributions with no splittings; however, splitting does become observable if the number of Ta sites included in the sum is reduced, so that the observation of a CDW splitting in this phase implies that there is no long-range coherence of the CDW's. With a finite coherence length, the $1: 6: 6$ distribution again approximately applies, but the peaks of the histogram are broadened; it is significant that the central feature, corresponding to $\alpha 2$, is always broadened more by deviations from commensurateness than is that corresponding to $\alpha 1$. Figure 2 shows that, even in the $1 \mathrm{~T}_{3}$ commensurate phase, $\alpha 2$ is always broader than $\alpha 1$, while both widths increase with temperature. This is consistent with the existence of local deviations from commensurateness i. e. there may exist short-range temperature dependent fluctuations of the CDW wavevectors in the commen- surate phase. Overhauser [13] has used the term phasons for local fluctuations of the CDW wavevectors. In the model calculations small deviations from the commensurate wavevectors always broaden $\alpha 2$ more than $\alpha 1$ for the $1: 6: 6$ distribution, and this is further evidence for this assignment. The greater width of $\alpha 2$ may also be partly attributable to the greater local charge gradient which this CDW configuration produces at the atoms contributing to the central feature of the $1: 6: 6$ distribution. The thermal motions of the two main types of atom would then produce a greater spread of chemical shifts for atoms in the greater charge gradient. This argument assumes however that the thermal motion of the lattice occurs under a rigid CDW charge distribution, which is clearly a weak approximation, but it is clear that local discrepancies between the CDW and the underlying lattice are responsible for the greater width of $\alpha 2$. Both widths increase markedly with the transformation to the quasi-commensurate $1 \mathrm{~T}_{2}$ phase, and exhibit hysteresis at this transition.

For the $1 \mathrm{~T}_{2}$ phase the repeat distance for stacking of the CDW's from layer to layer is three layers, so from electrostatic considerations rhombohedral stacking of the CDW's might be expected. However, this would require a shift of the $\mathrm{CDW}$ configuration from layer to layer, which would not produce the $1: 6: 6$ distribution observed, so it must be that intra-layer considerations dominate and the configurations for all layers are the same. Within this constraint, stacking is expected to be as close to rhombohedral as possible because of interlayer electrostatic effects.

Above $350 \mathrm{~K}$ in the truly incommensurate $1 \mathrm{~T}_{1}$ phase, no CDW splitting occurs, even in the second derivative. The $\mathrm{Ta} 4 \mathrm{f}$ lines are in fact narrower than at room temperature, where the CDW splitting, though not resolved, contributes substantially to the overall linewidths. So in the $1 \mathrm{~T}_{\mathbf{1}}$ phase, either the CDW's have achieved long-range coherence or the CDW amplitude has fallen so that the splitting is less than can be observed in this type of experiment.

The $\alpha 1-\alpha 2$ splitting as plotted in figure 2 is a combined measure of the CDW's amplitude and of their registration with the underlying $\mathrm{Ta}$ lattice, and in this sense represents an order parameter for the system. However there is still some ordering remaining in the $1 T_{1}$ phase where no splitting is observed, as the CDW amplitude is non-zero while the registration is lost.

The sulphur $2 p$ levels for this material show no CDW splitting and no MND tails, just sharpening slightly on cooling. This implies that the sulphur levels are comparatively unaffected by the conduction electrons, which can be presumed to be localized within the sheets of Ta atoms. In other words, the conduction electron states at the Fermi level have little anion admixture.

$2.21 \mathrm{~T}-\mathrm{TaSe}_{2}$. - The $\sqrt{13}$ commensurate phase of $1 \mathrm{~T}-\mathrm{TaSe}_{2}$ extends up to $473 \mathrm{~K}$ before the material 
transforms, without an intermediate phase, to the incommensurate phase, so all data reported here are on the commensurate phase. Even at $295 \mathrm{~K}$ a CDW splitting of the Ta $4 \mathrm{f}$ lines is clearly resolved (Fig. 3)

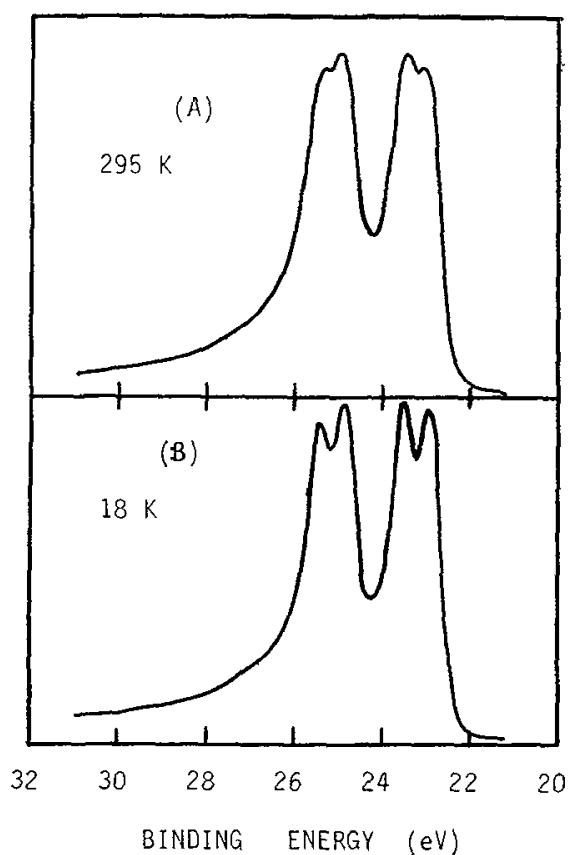

Fig. 3. - Ta $4 \mathrm{f}$ levels for $1 \mathrm{~T}-\mathrm{TaSe}_{2}$ at a) $295 \mathrm{~K}$, and b) $18 \mathrm{~K}$.

and at lower temperature it is clear that the spectra are closely similar to those for $1 \mathrm{~T}_{3}-\mathrm{TaS}_{2}$. The assignment of the same configuration, leading to a $1: 6: 6$ distribution of inequivalent $\mathrm{Ta}$ sites, is again appropriate, and all the discussion for $1 \mathrm{~T}_{3}-\mathrm{TaS}_{2}$ applies here.

The $\alpha 1-\alpha 2$ splitting and the widths of $\alpha 1$ and $\alpha 2$ are shown as a function of temperature in figure 4 . The CDW amplitude appears saturated at low temperature producing a splitting of $0.61 \mathrm{eV}$, and decreases slightly at higher temperatures. Again this represents a measure of the order of the CDW-lattice system although here the registration is perfect and only the CDW amplitude is changing. As before $\alpha 1$ and $\alpha 2$ become steadily broader with increasing temperature, with $\alpha 2$ always broader than $\alpha 1$, consistent with the hypothesis of short-range fluctuations. The Se $3 \mathrm{~d}$ levels show no CDW effects, just sharpening slightly on cooling as for the $\mathrm{S} 2 \mathrm{p}$ levels in $1 \mathrm{~T}-\mathrm{TaS}_{2}$.

$2.31 \mathrm{~T}^{-\mathrm{Ta}_{0.97}} \mathrm{Hf}_{0.03} \mathrm{~S}_{2}$. - $\mathrm{HfS}_{2}$ and $1 \mathrm{~T}-\mathrm{TaS}_{2}$ are isostructural, so apart from minor changes in lattice parameters, Hf-doped $1 \mathrm{~T}-\mathrm{TaS}_{2}$ retains its structure, with Hf substituting for Ta. This depletes the occupation of the $\mathrm{d}_{z^{2}}$ conduction band, and will tend to reduce the overall size of the Fermi surface and hence the CDW wavevectors. For $8.5 \%$ Hf doping the commensurate CDW state is suppressed [14] and at all accessible temperatures here it is assumed that an incommensurate state exists as in $1 \mathrm{~T}_{1}-\mathrm{TaS}_{2}$. As

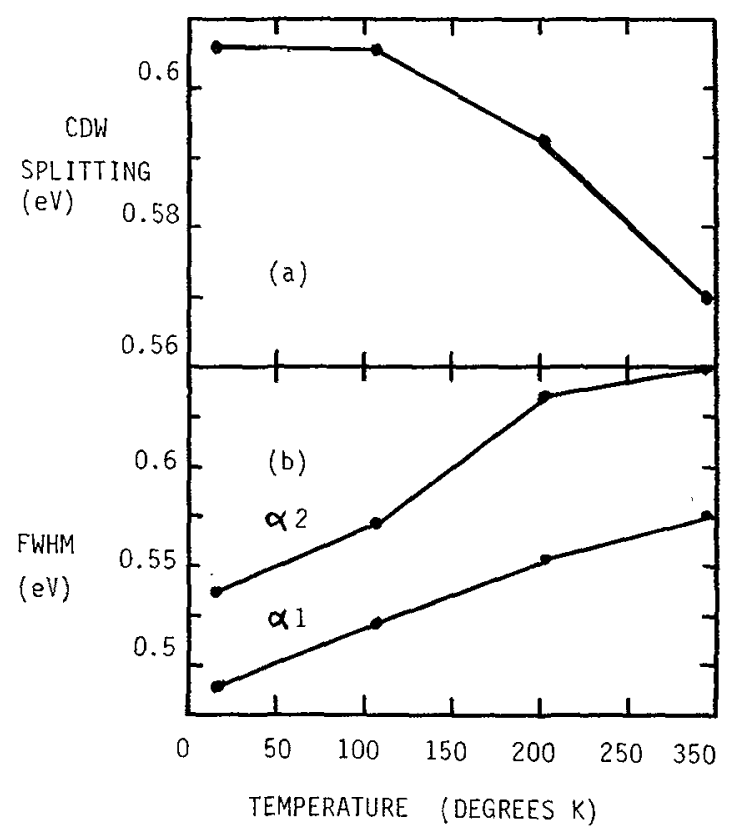

FIG. 4. - $1 \mathrm{~T}-\mathrm{TaSe}_{2}$ Ta $4 \mathrm{f} \frac{7}{2}$ line. a) CDW splitting, b) full widths at half maximum of components $\alpha 1$ and $\alpha 2$.

McMillan [15] points out, the incommensurate CDW is flexible and can distort in the impurity potential to lower its energy, stabilizing the incommensurate state relative to the commensurate.

Figure 5 shows the Ta $4 \mathrm{f} \frac{7}{2}$ line at $300 \mathrm{~K}$ and at $14 \mathrm{~K}$, and the Hf $4 \mathrm{f} \frac{7}{2}$ line (much weaker and less tightly bound) at $14 \mathrm{~K}$. A CDW splitting of $0.56 \mathrm{eV}$ occurs

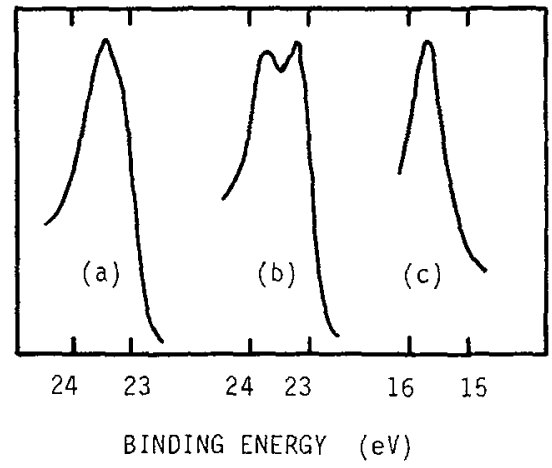

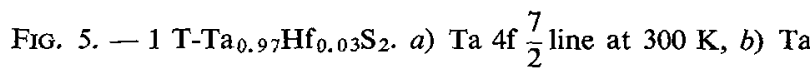
$4 \mathrm{f} \frac{7}{2}$ line at $14 \mathrm{~K}, c$ ) $\mathrm{Hf} 4 \mathrm{f} \frac{7}{2}$ line at $14 \mathrm{~K}$.

for the Ta line at $14 \mathrm{~K}$, and, by curve fitting with two peaks of equal intensity, can be obtained at all temperatures up to $300 \mathrm{~K}$, steadily decreasing to $0.37 \mathrm{eV}$. No effect occurs for the Hf lines, so while there are inequivalent Ta sites (as before consistent with a $1: 6: 6$ distribution), all Hf sites are equivalent. From the discussion earlier, an observable splitting for incom- 
mensurate CDW's implies short-range coherence, and it is likely that as all $\mathrm{Hf}$ sites have equivalent CDW charge environments that the Hf sites nucleate the CDW's in their vicinity, so that the coherence length is determined by the average Hf-Hf separation. For $3 \% \mathrm{Hf}$ : Ta ratio this is of the order of 6 atomic spacings. It seems likely that the Hf sites, with one fewer nuclear charge than the Ta sites, would attract the minima of the CDW charge density, which, in the absence of Hf, would correspond to the single site of the $1: 6: 6$ distribution.

$2.42 \mathrm{H}-\mathrm{TaSe}_{2}$. - The Ta 4 f spectra for $2 \mathrm{H}-\mathrm{TaSe}_{2}$ (Fig. 6) are quite different from those of $1 \mathrm{~T}-\mathrm{TaSe}_{2}$;

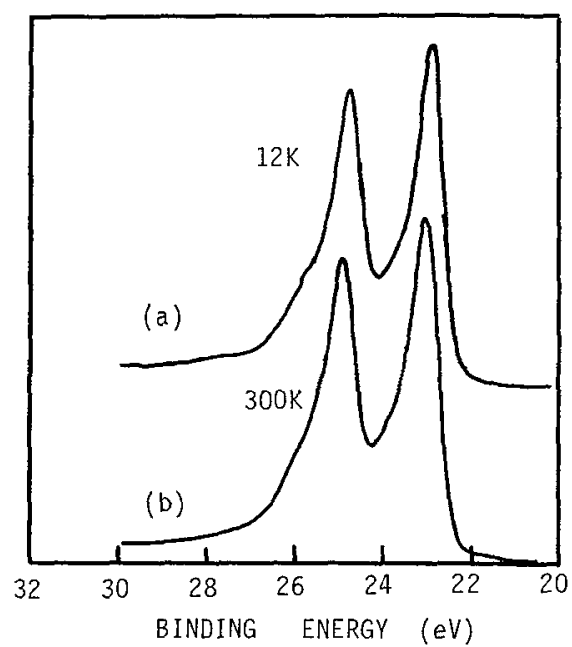

FIG. 6. - $2 \mathrm{H}-\mathrm{TaSe}_{2} \mathrm{Ta} 4 \mathrm{f}$ lines at a) $12 \mathrm{~K}$, and $b$ ) $300 \mathrm{~K}$.

the lines are sharper and the MND tails are apparently cut short so that a shoulder appears on the high binding energy side of each peak. This is probably a band structure effect, as, for the $2 \mathrm{H}$ polytype, a gap exists above the narrow $\mathrm{d}_{z^{2}}$ conduction band [16], so that a corresponding cut-off would occur in the electron-hole excitation spectrum. Virtually no change, apart from very slight sharpening, occurs on cooling to $14 \mathrm{~K}$; i. e. no $\mathrm{CDW}$ effects are observed even where it is established by electron and neutron diffraction that a commensurate $3 \times 3$ superlattice exists. The diffraction effects are, however, much weaker than in the case of, for instance, $1 \mathrm{~T}-\mathrm{TaSe}_{2}$, presumably because of the different Fermi surface geometry, so it is expected that all CDW effects would be far less pronounced.

3. Conclusion. - For the $1 \mathrm{~T}$ materials studied, these XPS measurements have permitted the different charge environments of different $\mathrm{Ta}$ atoms due to the presence of CDW's to be readily detected; this allows the spatial relationship between the CDW's and the underlying lattice, the configuration, to be deduced. The difference between inequivalent Ta atoms is most clear for the commensurate $\mathrm{CDW}$ condition. The temperature dependent broadening of the spectra for these commensurate phases indicates the existence of local discrepancies between the $\mathrm{CDW}$ system and the underlying lattice-local CDW fluctuations. Similar arguments extend to the quasi- and non-commensurate cases, where a CDW splitting should only be observed if the coherence of the CDW's is fairly short range ; for the quasi-commensurate phase of $1 \mathrm{~T}-\mathrm{TaS}_{2}$ this range is presumably determined by the spacing of defects, and by the spacing of $\mathrm{Hf}$ impurities in $1 \mathrm{~T}-\mathrm{Ta}_{0.97} \mathrm{Hf}_{0.03} \mathrm{~S}_{2}$. For the incommensurate $1 \mathrm{~T}_{1}$ phase of $1 \mathrm{~T}-\mathrm{TaS}_{2}$ no splitting is resolved, so either the CDW's have achieved long rage coherence or the CDW amplitude has fallen so much that the splitting is less than the instrumental resolution.

No effects are observed for $2 \mathrm{H}-\mathrm{TaSe}_{2}$ because of the much lower CDW amplitudes; this presumably is because of the different Fermi surface geometry for this material compared with the $1 \mathrm{~T}$ polytypes.

Acknowledgements. - Conversations with J. C. Tsang, W. M. Stobbs and M. W. Shafer (who also supplied the crystals) are gratefully acknowledged. We also thank Professor S. Meyer for a single crystal of $2 \mathrm{H}-\mathrm{TaSe}_{2}$, S. J. La Placa for structural determinations, and T. Penney and R. A. Craven for resistivity measurements.

\section{References}

[1] Wellams, P. M., Parry, G. S. and.Scruby, C. B., Phil. Mag. 29 (1974) 695-9.

[2] Wirson, J. A., di Salvo, F. J. and Mahajan, S., Phys. Rev. Lett. 32 (1974) 882-5.

[3] Wulson, J. A., di Salvo, F. J. and Mahajan, S., $A d v$. Phys. 24 (1975) 117-201.

[4] Scruby, C. B., Willuams, P. M. and Parry, G. S., Phil. Mag. 31 (1975) 255-74.

[5] Van landuyt, J., van Tendeloo, G. and Amelinckx, S., Phys. Stat. Sol. (a) 26 (1974) 359-76.

[6] Moncton, D. E., Axe, J. D. and DI SALvo, F. J., Phys. Rev. Lett. 34 (1975) 734-7.

[7] Wertheim, G. K., di Salvo, F. J. and Chiang, S., Phys. Lett. 54A (1975) 304-6.
[8] Hughes, H. P. and Pollak, R. A., Comm. Phys. 1 (1976) 61-5.

[9] Hughes, H. P. and Pollak, R. A., submitted for publication.

[10] Mahan, G. D., Phys. Rev. 163 (1967) 612-7.

[11] NoziÈres, C. and DE Dominicis, C. T., Phys. Rev. 178 (1969) 1097-1107.

[12] Doniach, S. and Sunjic, M., J. Phys. C : Solid State Phys. 3 (1970) 285-91.

[13] OVerhauser, A. W., Phys. Rev. B 3 (1971) 3173-82.

[14] di Salvo, F. J., Wirson, J. A., Bagley, B. G. and WaszcZAK, J. V., Phys. Rev. B 12 (1975) 2220-35.

[15] MCMillan, W. L., Phys. Rev. B 12 (1975) 1187-96 ; 1197-9.

[16] Mattheiss, L. F., Phys. Rev. B 8 (1973) 3719-40. 